\title{
Characteristics of Aperture Coupled Microstrip Antennas on Magnetized Ferrite Substrates
}

\author{
Germán León, Rafael R. Boix, Member, IEEE, Manuel J. Freire, and Francisco Medina, Senior Member, IEEE
}

\begin{abstract}
The method of moments in the spectral domain is applied to the full-wave analysis of aperture coupled microstrip antennas in the case where the substrate of the antennas is a layered medium containing magnetized ferrites. The subsectional basis functions used in the analysis make it possible to handle patch antennas and coupling apertures with right angle corners of arbitrary shape. The numerical results obtained show that antennas on ferrite substrates fed by single microstrip lines can radiate both circular and linear polarization, the polarization state being dependent on the orientation of the ferrites bias magnetic field. For a given polarization state, the matching frequency band of the antennas can be tuned over a wide frequency range by adjusting the magnitude of the bias magnetic field. Also, the polarization state can be continuously tuned from circular to linear within the same frequency band by adjusting both the magnitude and the orientation of the bias magnetic field. Some measurements are presented in order to check the validity of the numerical algorithm developed.
\end{abstract}

Index Terms-Magnetic tuning, microstrip antennas, microwave ferrites, polarization, spectral-domain approach.

\section{INTRODUCTION}

D UE to the recent availability of low loss commercial microwave ferrites, there is an increasing interest in studying the performance of microstrip antennas and microstrip circuits fabricated on magnetized ferrite substrates. In the case of microstrip antennas on ferrite substrates, these antennas present certain useful properties which are not found in conventional microstrip antennas fabricated on dielectrics. For instance, numerical and experimental results have shown that the operating frequency band of microstrip antennas on ferrites can be varied by adjusting the magnitude of the bias magnetic field [1]-[4]. Also, antennas printed on normally biased ferrites have been found to radiate circular polarization with one single feed [5], and the state of polarization has been found to be switchable between left-hand circular polarization (LHCP) and right-hand circular polarization (RHCP) as the direction of the bias magnetic field is reversed [3], [6]. In the case of square microstrip antennas on in-plane magnetized ferrites, two fundamental resonant modes can be excited which radiate orthogonal linear polarizations, but only one of these modes can be tuned by means of the bias magnetic field [7], [8]. Experimental results have

Manuscript received March 24, 2004; revised October 21, 2004. This work was supported by the Spanish Comisión Interministerial de Ciencia y Tecnología and European Union funds (FEDER) under Project TEC2004-03214.

The authors are with the Microwaves Group, Department of Electronics and Electromagnetism, College of Physics, University of Seville, 41012 Sevilla, Spain (e-mail: boix@us.es).

Digital Object Identifier 10.1109/TAP.2005.848470 shown that the polarization of these antennas can be tuned from circular to linear at a single frequency when the two resonant modes are excited simultaneously and the bias magnetic field is varied [7], [9]. Ferrite materials also show potential applications as substrates of the antennas of microstrip phased arrays since wide-angle impedance matching can be obtained by dynamically adjusting the bias magnetic field with scan angle [3], [10]. In addition, when the microstrip antennas of the phased arrays are fed by microstrip lines fabricated on a ferrite substrate, these feeding lines act as magnetically tunable phase shifters which make it possible to steer the main beam of the radiation pattern [4], [11]-[13]. Finally, several researchers have reported that the radar cross section of microstrip antennas fabricated on magnetized ferrites can be substantially reduced over a frequency range which can be tuned by means of the bias magnetic field (this is the frequency range where magnetostatic wave propagation is allowed along the ferrite substrates supporting the antennas) [14], [15].

According to Pozar's review paper on microstrip patch antennas [16], if the different techniques for feeding microstrip antennas are compared, the aperture coupled microstrip feed presents a few relevant advantages over the rest of techniques. The first advantage is that the aperture coupled microstrip feed makes it possible to use two independent substrates for the feeding network and the antenna element, which allows separate design of both the feeding and antenna functions [17], [18]. Also, the radiation arising from the feeding network cannot interfere with the main radiation pattern generated by the antenna since the ground plane separates the two radiation mechanisms [17]. A further advantage of these antennas is that the reactive part of the input impedance is controlled by the length of the open-end feeding microstrip line while the resistive part is controlled by the aperture length. Hence, there are two independent degrees of freedom for adjusting the input impedance of the antenna to a specified value [18]. Since the original aperture coupled microstrip antenna configuration was presented in [17] and [18], different versions of this configuration have been proposed in order to improve the antenna properties. For instance, wide-band aperture coupled antennas have been achieved by simply using a resonant aperture coupled to the resonant radiating patch [19], by stacking two coupled resonant patches [20], [21], or by using a combination of the two techniques [22]. Aperture coupled antennas with dual band performance have been realized with a single rectangular patch by cutting either slots or spur lines on the patch surface [23], [24]. Also, with a view to improving the coupling between the feeding lines and the radiating patches, the use of $\mathrm{H}$-shaped 
apertures [25] or bow-tie apertures [21] has been suggested as an alternative to standard rectangular apertures [18]. The existence of many different designs for aperture coupled antennas indicates that a flexible numerical algorithm for the analysis of these antennas should be able to handle radiating patches (single or stacked) and apertures of fairly arbitrary shapes [21], [23].

In this paper the authors apply the method of moments $(\mathrm{MoM})$ in the spectral domain to the full-wave analysis of aperture coupled antennas in the case where the radiating patches are printed on a multilayered substrate containing magnetized ferrite layers. Rooftop basis functions are used in the approximation of the electric current on the metallizations and the magnetic current of the apertures, which makes it possible to analyze aperture coupled antennas with apertures and patches of different shapes. Also, due to the use of suitable Green's functions in the spectral domain [26], the algorithm can deal with ferrite materials subjected to arbitrarily oriented bias magnetic fields. In previous papers dealing with the analysis of microstrip antennas on ferrite substrates, the MoM in the spectral domain has been applied to the study of proximity coupled antennas [27] and coaxial probe-fed antennas [3], the finite element-boundary integral method has been applied to the study of cavity-backed probe-fed antennas [28], and the TLM method has been applied to the study of contacting microstrip fed antennas [29]. To the authors' knowledge, the current paper is the first paper dealing with the study of aperture coupled microstrip antennas on ferrite substrates.

Concerning the contents of the paper, Section II briefly describes both the geometry of the antennas analyzed in the paper and the numerical procedure used for obtaining the input impedance and radiation fields of these antennas. Section III provides original numerical results for the matching and polarization characteristics of different aperture coupled antennas fabricated on magnetized ferrites. Emphasis is placed on studying how these matching and polarization characteristics are tuned by variations in the magnitude and the orientation of the bias magnetic field of the ferrites. Some of the numerical results presented are compared with measurements, and good agreement is found.

\section{PROBlem GeOMETRY AND OUTLINE OF THE NuMERICAL PROCEDURE}

Fig. 1(a) illustrates the side view of the type of aperture-coupled microstrip antenna treated in this paper. The radiating patch lies on a four-layered substrate containing three dielectric layers and one ferrite layer (some dielectric layers may not appear in the specific antennas analyzed in the results section, but the ferrite layer is always present), and the strip of the feeding microstrip line is printed on a single dielectric layer. Fig. 1(b) illustrates the orientation of the DC bias magnetic field existing inside the ferrite layer of Fig. 1(a) with respect to the coordinate axes defined in that figure. The numerical algorithm developed by the authors for the analysis of the antenna of Fig. 1(a) is capable of simulating patches and apertures with right angle corners of arbitrary shape. In particular, Fig. 1(c) and (d) illustrate the metallization lay-out and the dimensions of two

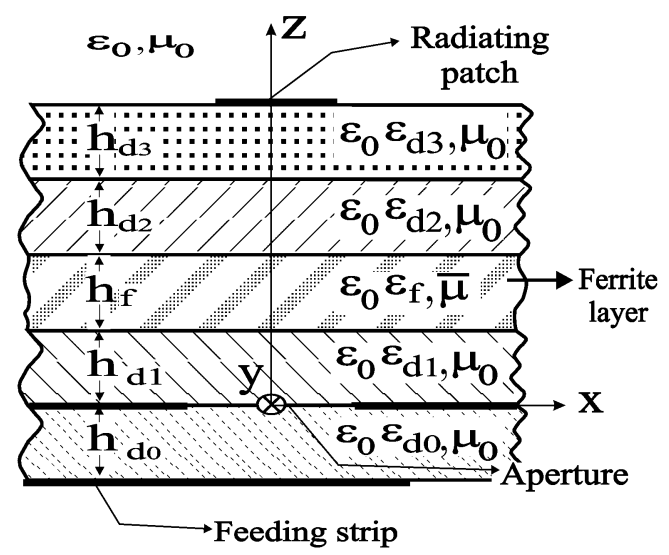

(a)

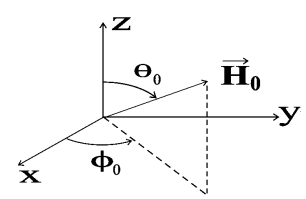

(b)

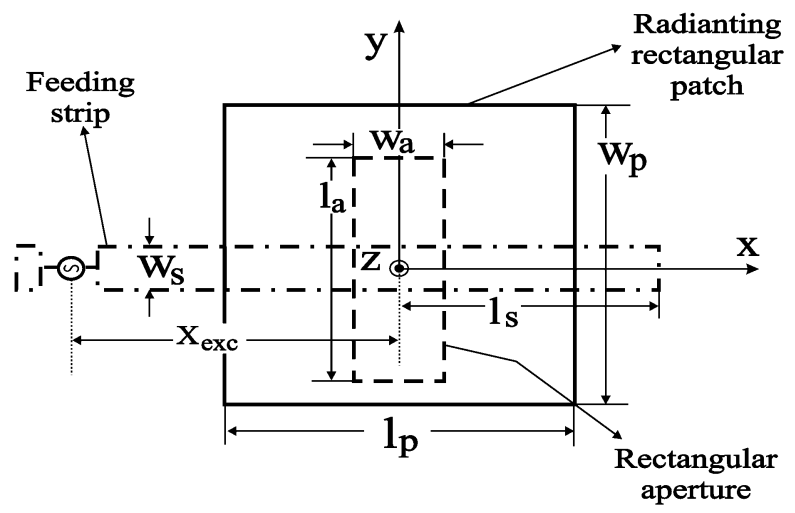

(c)

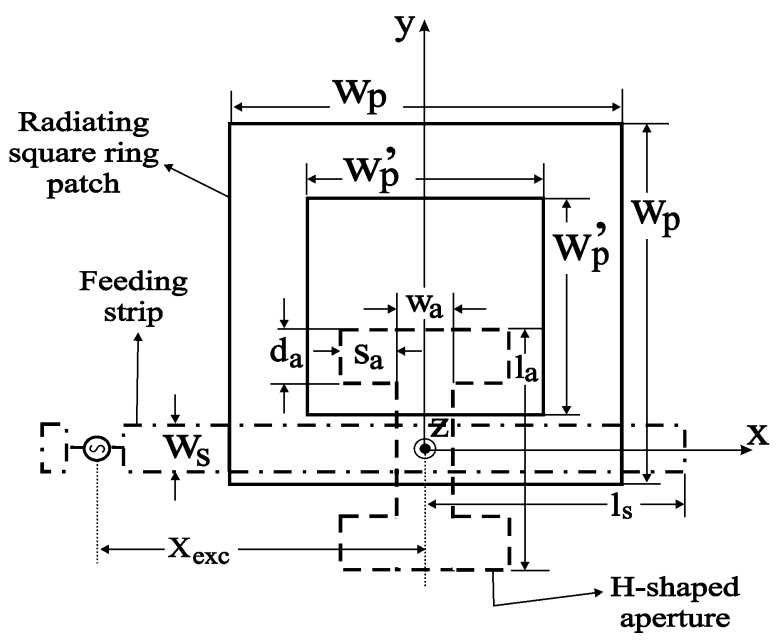

(d)

Fig. 1. (a) Side view of the aperture coupled microstrip antenna analyzed in this paper. (b) Orientation of the internal bias magnetic field of the ferrite layer with respect to the coordinate axes of (a). (c) Metallization lay-out of rectangular patch antenna coupled to a rectangular aperture. (d) Metallization lay-out of square ring patch antenna coupled to an $\mathrm{H}$-shaped aperture.

specific aperture-coupled antennas studied in the results section: a rectangular patch coupled to a rectangular aperture, and 
a square ring coupled to an $\mathrm{H}$-shaped aperture. As shown in Fig. 1(c) and (d), the microstrip line feeding the antenna is excited by a delta-gap voltage generator placed at the excitation plane $x=-x_{e x c}$. Note that the feeding microstrip line is left open at the excitation end as in [30].

Regarding the metallizations of Fig. 1(a) [see also Fig. 1(c) and (d)], in this paper the radiating patch, the ground plane containing the aperture and the strip of the feeding line are considered to be perfect electric conductors (PEC) of negligible thickness. Also, the ground plane as well as the dielectric and ferrite layers supporting the antenna are assumed to be of infinite extent along the $x$ and $y$ coordinates (e.g, the antenna is not cavity-backed [28]). The electromagnetic fields existing around the antenna of Fig. 1(a) have a time dependence of the type $e^{j \omega t}$, which will be assumed and suppressed throughout. Then, in general the permeability tensor of the ferrite layer can be written as

$$
\overline{\boldsymbol{\mu}}=\mu_{0}\left(\begin{array}{lll}
\mu_{x x} & \mu_{x y} & \mu_{x z} \\
\mu_{y x} & \mu_{y y} & \mu_{y z} \\
\mu_{z x} & \mu_{z y} & \mu_{z z}
\end{array}\right) .
$$

Throughout this work, the ferrite layer material will be assumed to be magnetically saturated. Under this assumption, the elements of the permeability tensor $\overline{\boldsymbol{\mu}}$ of (1) can be obtained in terms of the gyromagnetic ratio $\gamma=1.759 \cdot 10^{11} \mathrm{C} / \mathrm{Kg}$, the saturation magnetization of the ferrite material $M_{s}$, the magnitude of the internal bias magnetic field $H_{0}$, the linewidth of the ferrite material $\Delta H$, and the angles $\theta_{0}$ and $\phi_{0}$ of Fig. 1(b) as shown in [31, eqs. (1) to (4)] and in [32, eqs. (10.37) and (10.40)]. The magnitude of the externally applied magnetic field, $H_{e}$, and the angles giving the orientation of this external field with respect to the coordinate axes of Fig. 1(a) and (b), $\theta_{e}$ and $\phi_{e}$, can be obtained in terms of $M_{s}, H_{0}, \theta_{0}$ and $\phi_{0}$ by applying the boundary conditions for the tangential and normal magnetic field at the limiting planes of the ferrite layer (see [33, eqs. (1) and (2)]).

In order to obtain the circuit and radiation parameters of the aperture coupled antenna of Fig. 1(a), in this paper a set of three coupled integral equations has been obtained for the electric current density on the radiating patch, for the electric current density on the strip of the feeding line, and for the magnetic current density on the aperture. These three integral equations arise from enforcing the perfect electric conductor condition for the tangential electric field on both the patch and the strip of the feeding line, and from enforcing the continuity of the tangential magnetic field across the aperture [18], [21]. The Galerkin's version of the MoM has been used for solving the set of integral equations. Rooftop basis functions have been used in the approximation of the electric and magnetic current densities (see [30, eqs. (13) to (16)] and [34, eqs. (15) to (17)]), which restricts the analysis carried out to the case where the patch, the aperture and the metallic strip of Fig. 1(a) have right angle corners. A crucial point in the application of the MoM is that the Galerkin's matrix entries have all been computed in the spectral domain as in [35] ([35, eqs. (6), (8), and (9)] show how the spatial domain expressions of the entries are transformed into spectral domain expressions). The reason for working in the spectral domain is that whereas the computation of the dyadic Green's functions of the multilayered ferrite substrate of Fig. 1(a) is relatively straightforward in the spectral domain [26], [36], the same computation in the spatial domain is a extremely cumbersome task. As shown in [35], a drawback of the determination of the Galerkin's matrix entries in the spectral domain is that numerical computation of slowly convergent double infinite integrals is required. The authors have used special asymptotic extraction techniques for accelerating the computation of the infinite integrals (see the Section II and the Appendix of [35] for details), which has led to significant CPU time savings.

After solving the set of integral equations, the matrix of pencil technique has been used for de-embedding the antenna reflection coefficient $S_{11}$ in terms of the samples of the current on the feeding line [21], [30], [34]. Also, the two-dimensional Fourier transforms (2-D-FTs) of the tangential electric fields at the two limiting planes of the multilayered substrate of Fig. 1(a) have been obtained in terms of the 2-D-FTs of the electric and magnetic current densities via algebraic relations (see [26] and [36] for details). The 2-D-FTs of the tangential electric fields at the limiting planes have been used for obtaining the radiation electric field in the upper and lower half-spaces of Fig. 1(a) via the stationary phase method (see [37, pp. 164-169]). Once the radiation electric fields are known in the two half-spaces, antenna radiation parameters such as directivity, polarization state, and front-to-back ratio can be straightforwardly determined. The antenna gain has been determined in terms of the radiated electric field in the upper half space, and in terms of the power supplied to the antenna by means of the feeding microstrip line. This latter power has been calculated via the current existing along the microstrip line and via the characteristic impedance of the microstrip line (for details concerning the computation of the characteristic impedance, refer to [38]).

In order to reduce the computational expense arising from the analysis of the antenna of Fig. 1(a) over a large bandwidth, in this paper the Galerkin's matrix entries have been computed at a small number of frequencies within that interval, and closed form interpolating expressions have been generated which make it possible to compute the matrix entries at any frequency within the interval [3] (in the particular case of the antennas analyzed in Section III, numerical experiments have shown that the determination of sufficiently accurate interpolating expressions within a frequency interval of $1 \mathrm{GHz}$ only requires the numerical computation of the matrix entries at four different frequencies).

\section{NUMERICAL AND EXPERIMENTAL RESULTS}

The authors have written a numerical code for the analysis of aperture coupled microstrip antennas fabricated on ferrite substrates, which is based on the method described in Section II. In order to check the validity of this code, the results published in [18] for the input impedance of aperture coupled rectangular microstrip antennas fabricated on dielectrics have been compared with numerical results provided by our code in the nonmagnetic limit (i.e., the limit obtained when $M_{s} \rightarrow 0, H_{0} \rightarrow 0$ and $\Delta H \rightarrow 0$ inside the ferrite material). Also, numerical results supplied by the commercial package "Ensemble" for aperture coupled microstrip antennas of different shapes have been compared with our numerical results in the nonmagnetic limit. Good 
agreement has been found in all comparisons, but the results are not shown here for the sake of brevity.

Fig. 2(a) and (b) show results for an aperture coupled square patch. The patch is printed on a ferrite layer which is normally biased. The same patch on the same ferrite layer was analyzed by Pozar [3] by using coaxial probe feeding. Pozar observed that the patch had two close resonances which could radiate circularly polarized waves with one single feed. In fact, whereas the lower resonance radiated LHCP waves, the upper resonance radiated RHCP waves. Furthermore, the sense of polarization could be reversed by reversing the direction of the bias field. All these phenomena have also been observed for the square patch on ferrite substrate fed by a single microstrip line of Fig. 2(a) and (b). In fact, the results of Fig. 2(a) are for an antenna which can radiate LHCP waves (circular polarization is assumed when the axial ratio is smaller than $3 \mathrm{~dB}$ ), and the same antenna in Fig. 2(b) can radiate RHCP waves. If the antenna of Fig. 2(a) is assumed to be matched when $\left|S_{11}\right|<-10 \mathrm{~dB}$, the results of Fig. 2(a) show that the matching frequency range of this LHCP antenna can be tuned by $14 \%$ when the magnitude of the bias magnetic field is varied from $\mu_{0} H_{0}=0.01 \mathrm{~T}$ to $\mu_{0} H_{0}=$ $0.15 \mathrm{~T}$. Also, the matching frequency range of the RHCP antenna of Fig. 2(b) can be tuned by $8.5 \%$ when the magnitude of the bias field is varied from $\mu_{0} H_{0}=0.06 \mathrm{~T}$ to $\mu_{0} H_{0}=0.13 \mathrm{~T}$ (note that this tuning range is smaller than that obtained for the LHCP antenna since the matching of the RHCP antenna degrades as the magnitude of the bias field recedes from $\mu_{0} H_{0}=$ $0.1 \mathrm{~T}$ ). These tuning capabilities were also commented by Pozar [3]. The problem with the antenna of Fig. 2(a) and (b) is that the bandwidth of the antenna for every value of $\mu_{0} H_{0}$ is so small (less than $1 \%$ in the LHCP case and less than $0.5 \%$ in the RHCP case) that the frequency range in which the antenna is matched practically never coincides with the frequency range in which the axial ratio is below $3 \mathrm{~dB}$ (the situation is especially bad in the RHCP case), which negates the use of this antenna for circular polarization applications.

Bearing in mind the poor performance of the circularly polarized antenna of Fig. 2(a) and (b), in Fig. 3(a) and (b), a new design is proposed where the ferrite layer supporting the patch is separated from the ground plane by means of a foam layer. The presence of the foam layer not only increases the bandwidth of the antenna for every value of $\mu_{0} H_{0}$ (about $6 \%$ in the LHCP case and about $1.5 \%$ in the RHCP case) but also helps to close the gap between the matching frequency range and the $3 \mathrm{~dB}$ axial ratio frequency range. In fact, in Fig. 3(a) and (b) the axial ratio is always below $3 \mathrm{~dB}$ when $\left|S_{11}\right|<-10 \mathrm{~dB}$. The presence of the foam layer also improves the gain, which is about $4.5 \mathrm{~dB}$ in the antenna of Fig. 2(a) and (b), and about $7 \mathrm{~dB}$ in the antenna of Fig. 3(a) and (b). In addition, the front-to-back ratio is slightly better in the antenna of Fig. 3(a) and (b) (about $21 \mathrm{~dB}$ ) than in the antenna of Fig. 2(a) and (b) (about $17 \mathrm{~dB}$ ). The LHCP antenna of Fig. 3(a) can be tuned by $9.5 \%$ while the RHCP antenna of Fig. 3(b) can be tuned by $19 \%$. Note that the tuning range of the LHCP antenna is substantially smaller than that of the RHCP antenna. The tuning values of $\mu_{0} H_{0}$ have to be limited in order to avoid the excitation of magnetostatic volume waves (MSVWs) [39] along the ferrite substrate in the operating frequency band of the antenna (when magnetostatic waves are

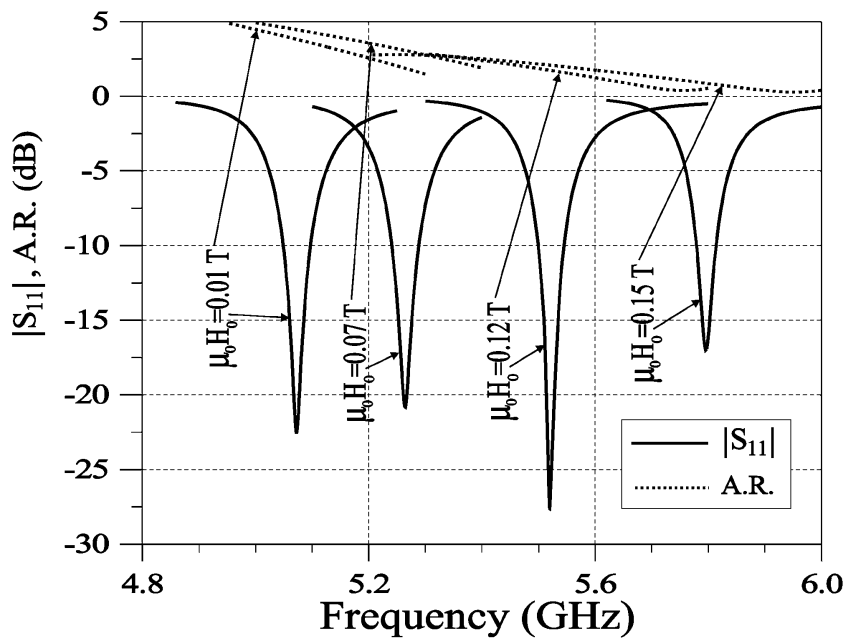

(a)

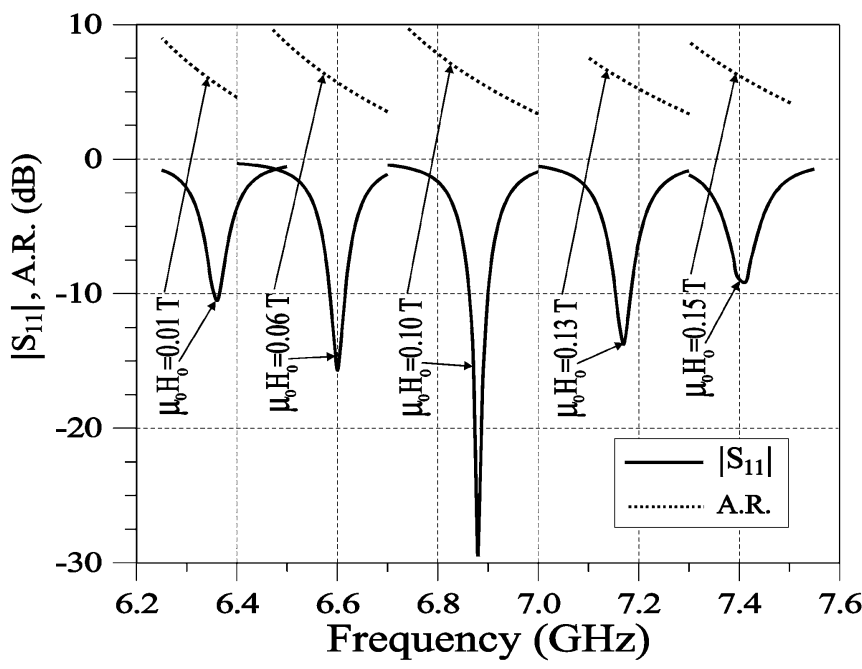

(b)

Fig. 2. Reflection coefficient and axial ratio of square microstrip patch coupled to a rectangular aperture [see Fig. 1(c)]. The patch substrate is a single ferrite layer. The ferrite is normally biased $\left(\theta_{0}=0^{\circ}\right)$ and two different resonant modes are studied leading to LHCP (a) and RHCP (b). Results are presented for different values of the magnitude of the bias magnetic field $H_{0}$. (a) and (b): $h_{d 0}=0.635 \mathrm{~mm}, h_{f}=1.27 \mathrm{~mm}, h_{d 1}=h_{d 2}=h_{d 3}=0, \epsilon_{d 0}=10$, $\epsilon_{f}=15, \mu_{0} M_{s}=0.065 \mathrm{~T}, \Delta H=0, w_{s}=0.6 \mathrm{~mm}, w_{a}=0.4 \mathrm{~mm}$, $w_{p}=l_{p}=6.1 \mathrm{~mm} ;(\mathrm{a}): l_{a}=5 \mathrm{~mm}, l_{s}=1.85 \mathrm{~mm}$; (b): $l_{a}=4 \mathrm{~mm}$, $l_{s}=1.2 \mathrm{~mm}$.

excited, they capture the power supplied to the antenna, and this has a deleterious effect on the antenna gain as shown in [14]), and the maximum allowed value of $\mu_{0} H_{0}$ for the LHCP antenna of Fig. 3(a) $\left(\mu_{0} H_{0}=0.09 \mathrm{~T}\right)$ turns out to be smaller than that for the RHCP antenna of Fig. 3(b) $\left(\mu_{0} H_{0}=0.135 \mathrm{~T}\right)$. It should be pointed out that the results plotted in Fig. 3(a) and (b) are directly related to the square patch LHCP and RHCP resonances placed above the frequency range of MSVWs (this frequency range is $\mu_{0} \gamma H_{0} / 2 \pi<f<\mu_{0} \gamma \sqrt{H_{0}\left(H_{0}+M_{s}\right)} / 2 \pi$ [39], and coincides with the frequency range where the ferrite permeability tensor element $\mu$ defined in [32, eqn. (10.25a)] is negative). However, the square patch on normally biased ferrite also presents LHCP and RHCP resonances below the range of MSVWs. These resonances, which do not appear in Fig. 2 of [3], were reported in Fig. 3 of [40] for a circular patch printed on a ferrite substrate. The authors of this paper have been able to find 


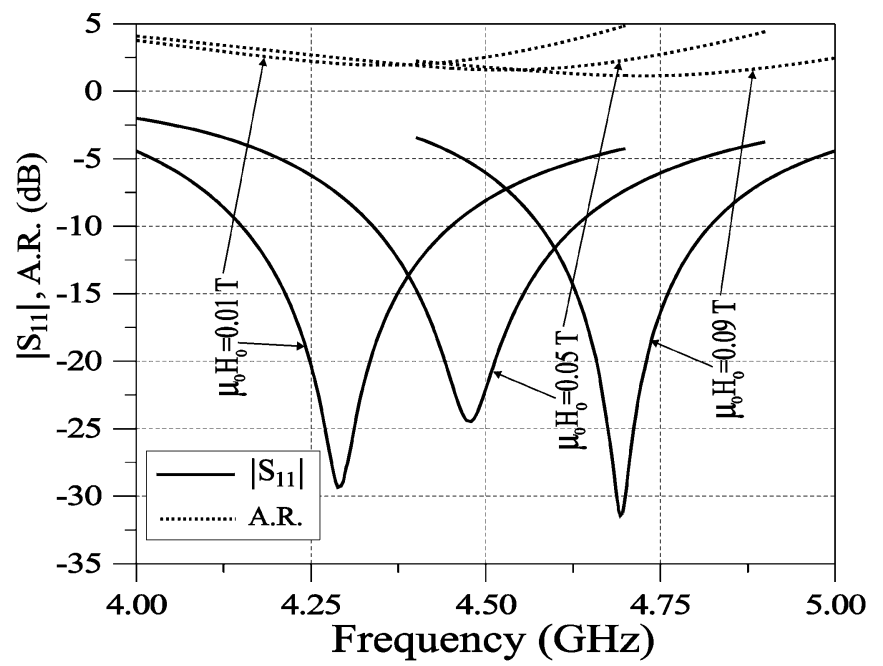

(a)

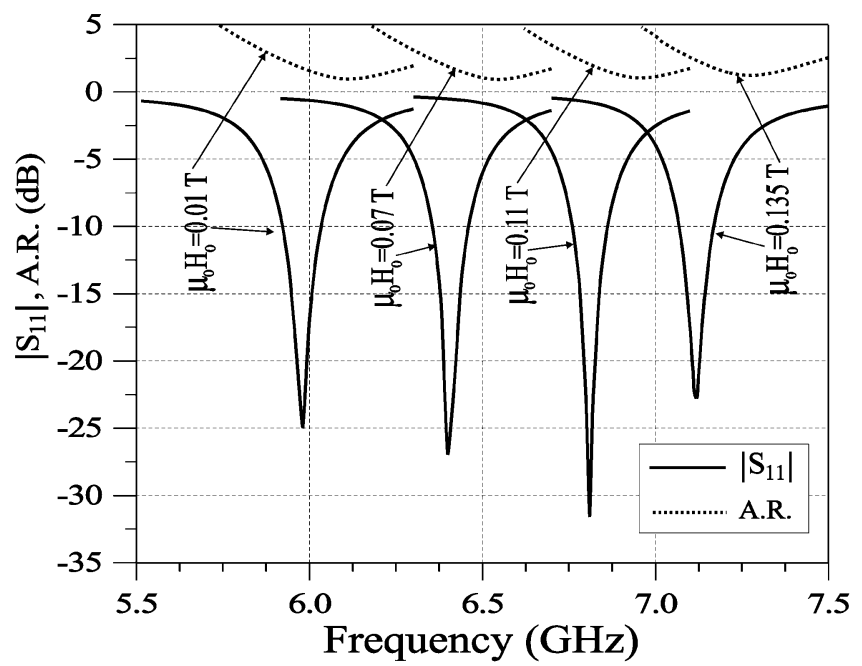

(b)

Fig. 3. Reflection coefficient and axial ratio of square microstrip patch coupled to a rectangular aperture [see Fig. 1(c)]. The patch lies on double-layered ferrite-foam substrate. The ferrite is normally biased $\left(\theta_{0}=0^{\circ}\right)$ and two different resonant modes are studied leading to (a) LHCP and (b) RHCP. Results are presented for different values of the magnitude of the bias magnetic field $H_{0}$. (a) and (b): $h_{d 0}=0.635 \mathrm{~mm}, h_{d 1}=h_{f}=1.27 \mathrm{~mm}$, $h_{d 2}=h_{d 3}=0, \epsilon_{d 0}=10, \epsilon_{d 1}=1.07, \epsilon_{f}=15.3, \mu_{0} M_{s}=0.183 \mathrm{~T}$, $\mu_{0} \Delta H=0.0006 \mathrm{~T}, w_{s}=0.6 \mathrm{~mm}, w_{a}=0.4 \mathrm{~mm}, w_{p}=l_{p}=11.5 \mathrm{~mm}$; (a): $l_{a}=12.2 \mathrm{~mm}, l_{s}=4 \mathrm{~mm}$; (b) $: l_{a}=9 \mathrm{~mm}, l_{s}=5.9 \mathrm{~mm}$.

the LHCP and RHCP resonances below MSVWs for the square patch analyzed in Fig. 3(a) and (b). Unfortunately, these two resonances are not useful for circularly polarized antenna applications. The reason for this is that the two resonant frequencies are very close to each other, which leads to a quick variation of the axial ratio around the resonances. As a consequence of this fact, it is very challenging to overlap the matching frequency range of the antenna with the $3 \mathrm{~dB}$ axial ratio frequency range, as it happens with the antenna analyzed in Fig. 2(a) and (b).

Tsang et al. have published experimental results that demonstrate that annular ring patches on normally biased ferrite substrates also radiate LHCP and RHCP waves [6]. Since both square patches and annular ring patches radiate LHCP and RHCP waves, the authors of this paper decided to investigate if square ring patches on normally biased ferrites are able to radiate LHCP and RCHP waves. Also, in order to show that the numerical algorithm described in Section II is able to handle patches and apertures of many different shapes, the square ring patches were fed by a microstrip line via an $\mathrm{H}$-shaped aperture [see Fig. 1(d)]. The results obtained for the aperture coupled square ring antenna are shown in Fig. 4(a) and (b). The substrate of the antenna is the same substrate used for the antenna of Fig. 3(a) and (b). Note that the results of Fig. 4(a) and (b) do not substantially differ from those of Fig. 3(a) and (b), which proves that the square ring patch on normally biased ferrite can also be used for radiating LHCP and RCHP waves. As it happens with Fig. 3(a) and (b), in Fig. 4(a) and (b) the axial ratio is always below $3 \mathrm{~dB}$ in the frequency range where matching is achieved. The bandwidths obtained for every value of $\mu_{0} H_{0}$ in Fig. 4(a) and (b) (about 5.5\% in the LHCP case and about $1 \%$ in the RHCP case) are slightly smaller than those obtained in Fig. 3(a) and (b), which agrees with the fact that the quality factors of the square ring resonances computed via the method of [35] are slightly larger than those of the square patch resonances. The gain, front-to-back ratio and magnetic tuning range of the antenna of Fig. 4(a) and (b) are similar to those of the antenna of Fig. 3(a) and (b). The only advantage of the antenna of Fig. 4(a) and (b) over the antenna of Fig. 3(a) and (b) is that it operates in similar frequency bands with a smaller size.

In Figs. 3 and 4 we have studied circularly polarized antennas with a single feed which are able to provide a fairly large bandwidth (between $15 \%$ and 20\%) for both matching and axial ratio with the help of a continuously adjusting magnetic field. However, it would be more convenient to have a design of circularly polarized antenna on ferrite substrate with one single feed and large bandwidth which is biased by a constant magnetic field. The authors have found an antenna design of the aforementioned type (i.e., only requiring a constant magnetic field), which is shown in Fig. 5(a) and (b). The antenna consists of two stacked square patches, which is a typical configuration for bandwidth improvement [20], [21]. The two patches lie on ferrite layers, and the ferrite layers are separated by a foam layer. The ferrite layers are normally biased by a constant magnetic field. Although high permittivity substrates are not very adequate for microstrip antennas [16], Waterhouse recently proved that the combination of high permittivity and low permittivity substrates in stacked patch antennas leads to good antenna performance [41]. Bearing in mind this idea, the authors designed a stacked patch antenna in which the lower patch is printed on a ferrite layer with high permittivity and the upper patch rests on a two-layered ferrite-foam medium with lower effective permittivity [see Fig. 5(a)]. The results obtained for the antenna are shown in Fig. 5(c). The antenna presents a 31\% bandwidth for $\left|S_{11}\right|<-15 \mathrm{~dB}$, and the axial ratio for LHCP radiation is below $3 \mathrm{~dB}$ in the whole matching frequency range. The gain (in fact, the relative gain is plotted in Fig. 5(c) including mismatch losses) is between 4 and $5 \mathrm{~dB}$ in most of the operating frequency band of this single-feed antenna. Similar designs for circularly polarized aperture coupled antennas on dielectric substrates with one single feed lead to bandwidths that are substantially smaller [42]. And wideband circularly polarized aperture coupled antennas on dielectric substrates are only achieved at 


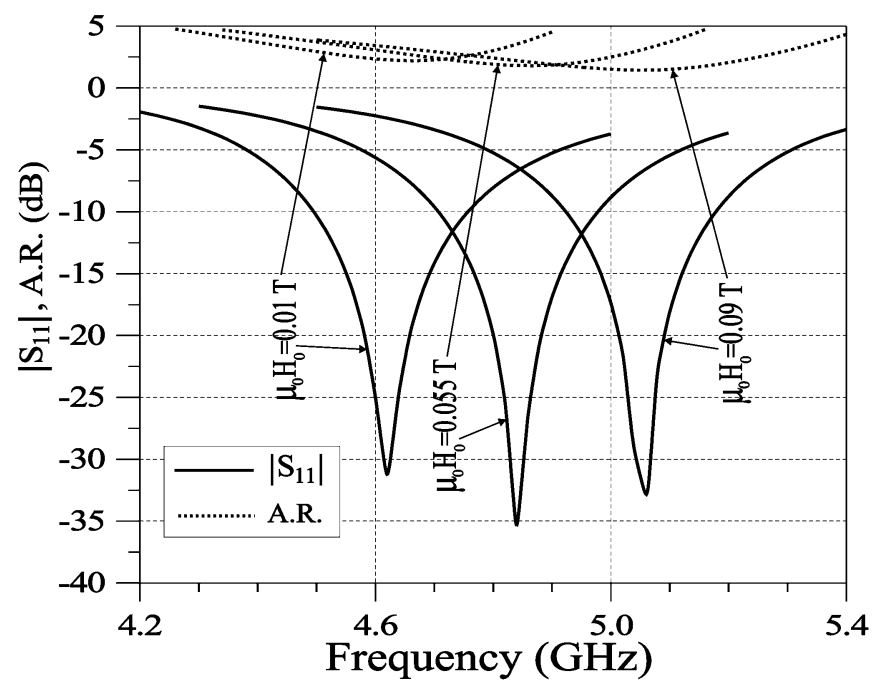

(a)

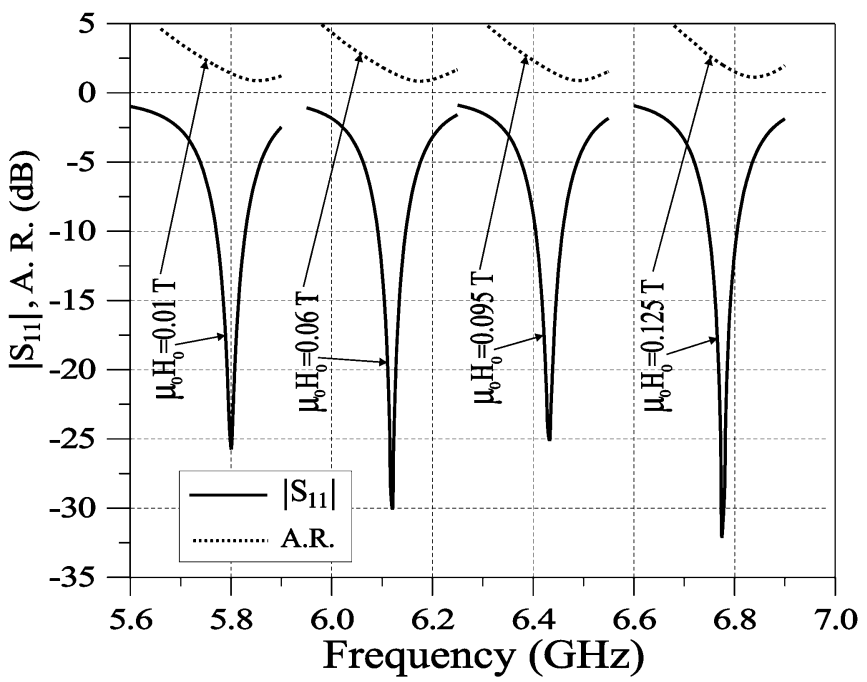

(b)

Fig. 4. Reflection coefficient and axial ratio of square ring microstrip patch coupled to an $\mathrm{H}$-shaped aperture [see Fig. 1(d)]. The patch lies on double-layered ferrite-foam substrate. The ferrite is normally biased $\left(\theta_{0}=0^{\circ}\right)$ and two different resonant modes are studied leading to LHCP (a) and RHCP (b). Results are presented for different values of the magnitude of the bias magnetic field $H_{0}$. (a) and (b): $h_{d 0}=0.635 \mathrm{~mm}, h_{d 1}=h_{f}=1.27 \mathrm{~mm}$, $h_{d 2}=h_{d 3}=0, \epsilon_{d 0}=10, \epsilon_{d 1}=1.07, \epsilon_{f}=15.3, \mu_{0} M_{s}=0.183 \mathrm{~T}$, $\mu_{0} \Delta H=0.0006 \mathrm{~T}, w_{s}=0.6 \mathrm{~mm}, w_{a}=0.4 \mathrm{~mm}, s_{a}=0.8 \mathrm{~mm}$, $w_{p}=9.5 \mathrm{~mm}, w_{p}^{\prime}=4.75 \mathrm{~mm}$; (a): $d_{a}=0.84 \mathrm{~mm}, l_{a}=8.4 \mathrm{~mm}$, $l_{s}=3 \mathrm{~mm} ;(\mathrm{b}): d_{a}{ }^{p}=0.75 \mathrm{~mm}, l_{a}=6 \mathrm{~mm}, l_{s}=5.67 \mathrm{~mm}$.

the expense of using very sophisticated feeding networks [43]. It should be mentioned that the numerical algorithm required for the analysis of the antenna of Fig. 5(a) and (b) is an extension of the numerical algorithm described in Section II (an additional integral equation is needed to account for the second radiating patch [21]).

In Fig. 6(a) and (b), the authors analyze an aperture coupled square patch antenna on two-layered ferrite-foam substrate (i.e., the substrate of the antennas of Figs. 3 and 4) in the case where the ferrite is biased in-plane in a direction parallel to two of the sides of the square patch. The square patch has two main resonances with orthogonal currents above the range of excitation of magnetostatic waves (REMW) and two other main resonances with the same orthogonal current pattern below the

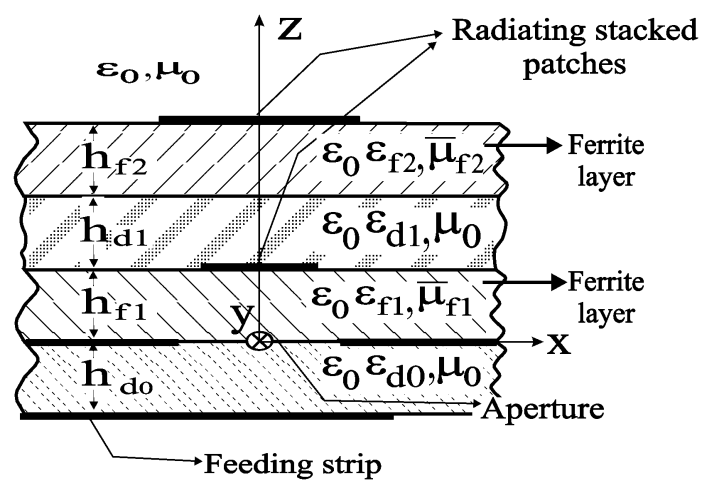

(a)

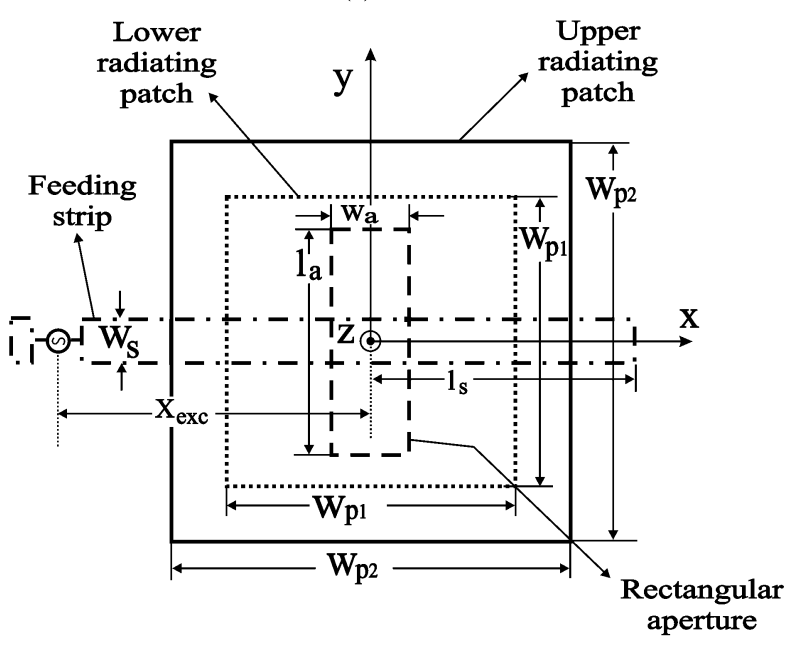

(b)

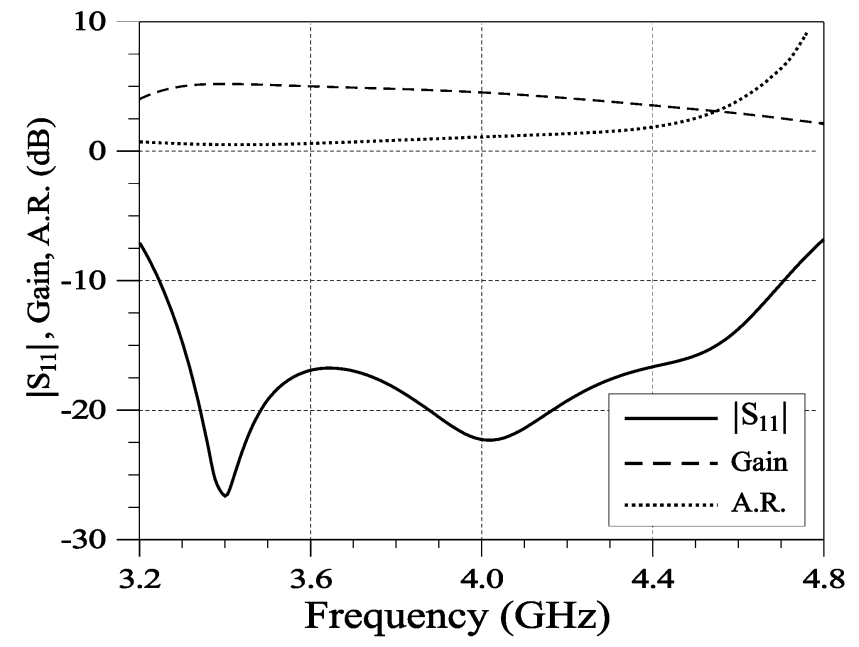

(c)

Fig. 5. (a) Side view of aperture coupled stacked microstrip antenna fabricated on multilayered substrate containing ferrite layers. (b) Metallization lay-out of the aperture coupled antenna of (a) containing two stacked square patches on top of a rectangular aperture. (c) Reflection coefficient, gain and axial ratio of the antenna of (a) and (b) in the case where the ferrite layers are normally biased $\left(\theta_{0}=0^{\circ}\right) . h_{d 0}=0.635 \mathrm{~mm}, h_{f 1}=h_{f 2}=1.27 \mathrm{~mm}, h_{d 1}=3.175 \mathrm{~mm}$, $\epsilon_{d 0}=10, \epsilon_{f 1}=\epsilon_{f 2}=15.3, \mu_{0} M_{s 1}=\mu_{0} M_{s 2}=0.183 \mathrm{~T}, \mu_{0} \Delta H_{1}=$ $\mu_{0} \Delta H_{2}=0.0006 \mathrm{~T}, \mu_{0} H_{01}=\mu_{0} H_{02}=0.04 \mathrm{~T}, \epsilon_{d 1}=1.07, w_{s}=$ $0.6 \mathrm{~mm}, l_{s}=8 \mathrm{~mm}, w_{a}=0.4 \mathrm{~mm}, l_{a}=13 \mathrm{~mm}, w_{p 1}=9 \mathrm{~mm}, w_{p 2}=$ $12 \mathrm{~mm}$.

REMW (these four resonances are those of the resonant modes $m=0, n=1$ and $m=1, n=0$ of Fig. 4 of [35]). All four resonances lead to linearly polarized radiation. In the case of the 
two resonances with currents perpendicular to the bias magnetic field (one above the REMW and one below), the resonant frequencies are not appreciably affected by variations in the magnitude of the magnetic field [8], [35]. However, in the case of the two resonances with currents parallel to the bias magnetic, the resonant frequencies substantially change as the magnitude of the bias field is varied [8], [35]. These latter two resonances are those studied in Fig. 6(a) and (b). Fig. 6(a) is devoted to the resonance above the REMW and Fig. 6(b) is devoted to the resonance below. The antenna above the REMW can be tuned by $10 \%$ as $\mu_{0} H_{0}$ is varied, and the antenna below the REMW can be tuned by $15 \%$. In the case of the antenna above the REMW of Fig. 6(a), the magnitude of the tuning magnetic field has to be limited because of the excitation of magnetostatic surface waves (MSSWs) [39] along the ferrite substrate, and because of the excitation of strip-guided magnetostatic surface wave resonances [44]. These two phenomena have a deleterious effect on the antenna gain and the frequency range where they appear should be avoided as much as possible (this frequency range is $\mu_{0} \gamma \sqrt{H_{0}\left(H_{0}+M_{s}\right)} / 2 \pi<f<\mu_{0} \gamma\left(H_{0}+M_{s}\right) / 2 \pi$ [39], [44], and coincides with the frequency range where the ferrite effective permeability $\mu_{e}$ defined in [32, eq. (10.65)] is negative). In the case of the antenna below the REMW of Fig. 6(b), the value of $\mu_{0} H_{0}$ cannot be varied over a wide range because matching degrades as $\mu_{0} H_{0}$ recedes from $0.15 \mathrm{~T}$. The bandwidth of the antenna for every value of $\mu_{0} H_{0}$ is about $2.5 \%$ above the REMW, and about $4.5 \%$ below the REMW. The radiation pattern of the antenna above the REMW is more directive than that of the antenna below the REMW, and as a consequence of this, the gain of the antenna above the REMW (about $9 \mathrm{~dB}$ ) is larger than that of the antenna below the REMW (about $4.75 \mathrm{~dB}$ ). The front to back ratio is above $20 \mathrm{~dB}$ both above and below the REMW.

Since square microstrip antennas on normally biased ferrites radiate circular polarization [as shown in Figs. 3(a) and (b) and 5(c)] and square microstrip antennas on in-plane biased ferrites radiate linear polarization (this is the case of the antenna of Fig. 6(a) and (b)), it seems that the polarization of a square microstrip antenna on ferrite substrate could be tuned from circular to linear by rotating the orientation of the bias magnetic field from normal direction to in-plane direction. However, in order to tune the polarization of the antenna on ferrite substrate within the same operating frequency band, it is also necessary to change the magnitude of the internal bias magnetic field. And this is because the resonant frequencies of the microstrip antenna on normally and in-plane biased ferrites are different for the same magnitude of the internal bias magnetic field, which makes it unavoidable to vary the magnitude of the internal bias field if the antenna on normally biased ferrite has to operate at the same frequency as the antenna on in-plane biased ferrite. Also, there is an additional source of change for the external magnetic field since the demagnetization factor relating the external magnetic field and the internal magnetic field $H_{0}$ varies from a normally biased ferrite layer to an in-plane biased ferrite layer as shown in [32]. In Fig. 7(a), the authors study an aperture coupled square patch antenna on ferrite substrate whose polarization is tuned by rotating the orientation of the bias field in a plane perpendicular to the axis of the feeding microstrip line. The same study is carried out in Fig. 7(b) for the case where the

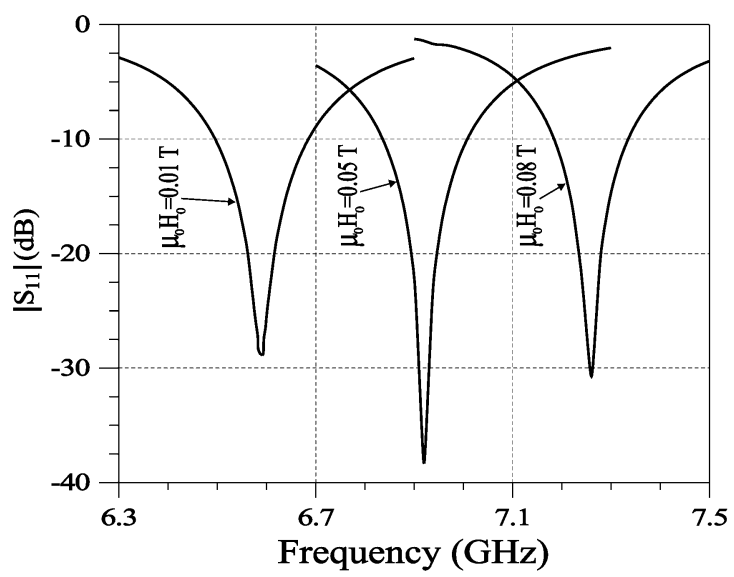

(a)

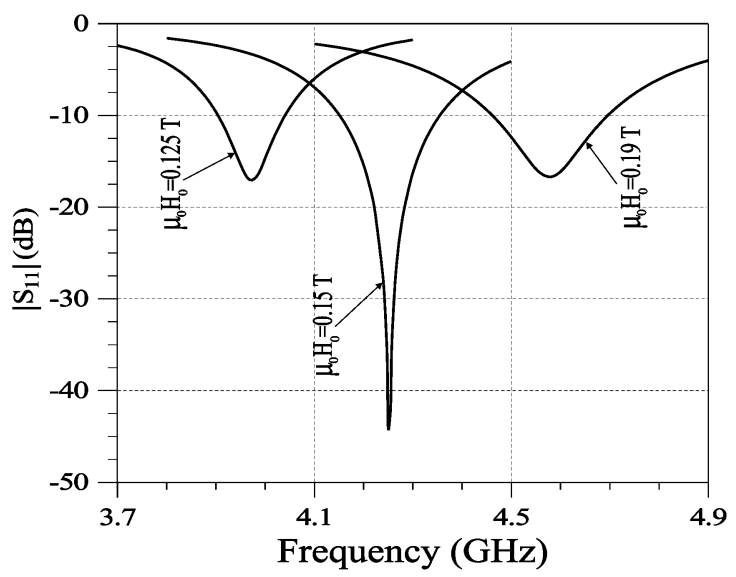

(b)

Fig. 6. Reflection coefficient of square microstrip patch coupled to a rectangular aperture [see Fig. 1(c)]. The patch lies on double-layered ferrite-foam substrate. The ferrite is biased in-plane $\left(\theta_{0}=90^{\circ}, \phi_{0}=0^{\circ}\right)$. Results are presented for different values of the magnitude of the bias magnetic field $H_{0}$ above (a) and below (b) the REMW. (a) and (b): $h_{d 0}=0.635 \mathrm{~mm}$, $h_{d 1}=h_{f}=1.27 \mathrm{~mm}, h_{d 2}=h_{d 3}=0, \epsilon_{d 0}=10, \epsilon_{d 1}=1.07, \epsilon_{f}=15.3$, $\mu_{0} M_{s}=0.183 \mathrm{~T}, \mu_{0} \Delta H=0.0006 \mathrm{~T}, w_{s}=0.6 \mathrm{~mm}, w_{a}=0.4 \mathrm{~mm}$ $w_{p}=l_{p}=8.5 \mathrm{~mm} ;(\mathrm{a}): l_{a}=8 \mathrm{~mm}, l_{s}=5.33 \mathrm{~mm}$; (b): $l_{a}=12 \mathrm{~mm}$, $l_{s}=5.33 \mathrm{~mm}$.

polarization is tuned by rotating the orientation of the bias field in a plane parallel to the the axis of the feeding microstrip line. In the two figures the magnitude of the internal bias field changes with the orientation with a view to keeping a single frequency range in which the antenna is matched for both linear and circular polarization. In Fig. 7(a) the matching frequency range lies between 5.26 and $5.41 \mathrm{GHz}$, and in Fig. 7(b) the matching frequency range lies between 6.75 and $6.92 \mathrm{GHz}$. When the orientation of the internal bias field makes an angle of $45^{\circ}$ with respect to broadside, in Fig. 7(a) the polarization is elliptic with axial ratio around $6 \mathrm{~dB}$ but in Fig. 7(b) the polarization is still RHCP (axial ratio below $3 \mathrm{~dB}$ ). However, when the ferrite is biased in-plane $\left(\theta_{0}=90^{\circ}\right)$ the polarization turns out to be linear both in Fig. 7(a) and (b). It should be pointed out that the antenna gain and the front-to-back ratio remain practically constant both in Fig. 7(a) and (b) as the orientation and magnitude of the internal bias field are changed for tuning the polarization.

Fig. 8(a) and (b) compare numerical and experimental results for the reflection coefficient of an aperture coupled square patch antenna. This antenna is supported by a multilayered substrate 


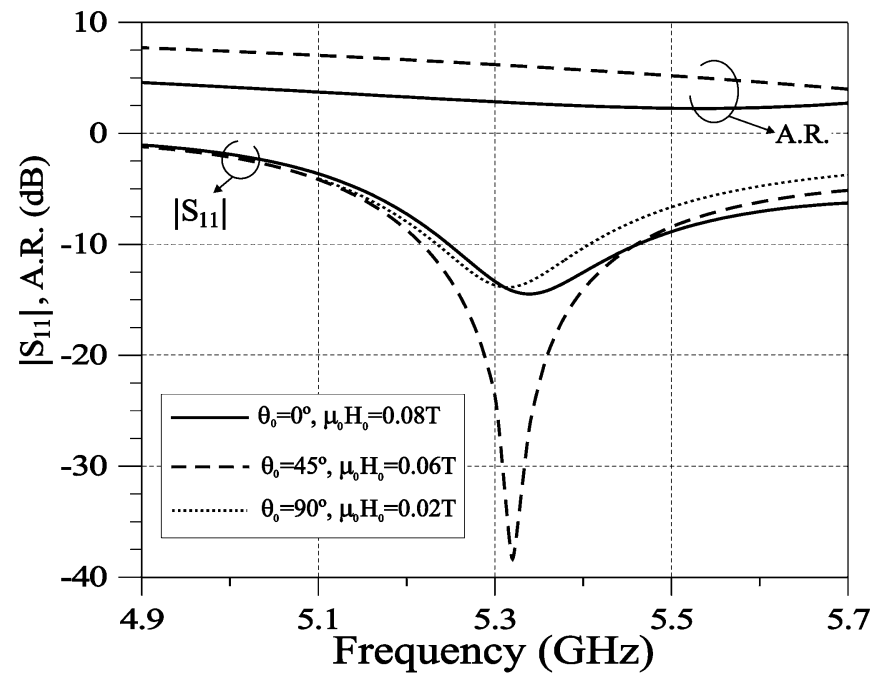

(a)

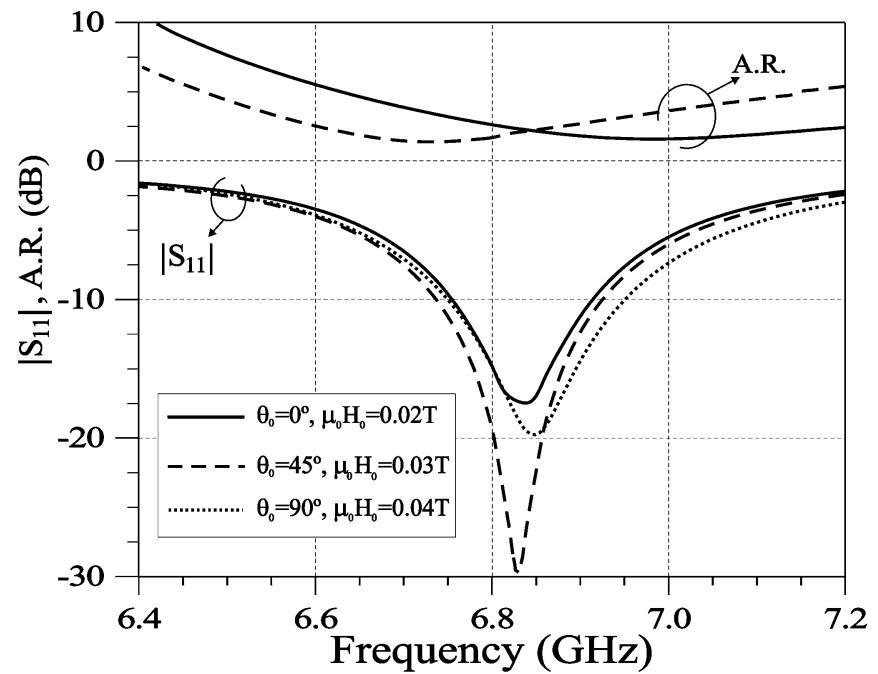

(b)

Fig. 7. Reflection coefficient and axial ratio of square microstrip patch coupled to a rectangular aperture [see Fig. 1(c)]. The patch lies on double-layered ferrite-foam substrate. Results are presented for different orientations $\left[\phi_{0}=90^{\circ}\right.$ in (a) and $\phi_{0}=0^{\circ}$ in (b) $]$ and magnitudes of the bias magnetic field of the ferrite. The polarization state obtained when the ferrite is normally biased $\left(\theta_{0}=0^{\circ}\right)$ is LHCP in (a) and RHCP in (b). (a) and (b): $h_{d 0}=0.635 \mathrm{~mm}, h_{d 1}=h_{f}=1.27 \mathrm{~mm}, h_{d 2}=h_{d 3}=0$, $\epsilon_{d 0}=10, \epsilon_{d 1}=1.07, \epsilon_{f}=15.3, \mu_{0} M_{s}=0.183 \mathrm{~T}, \mu_{0} \Delta H=0.0006 \mathrm{~T}$, $w_{s}=0.6 \mathrm{~mm}, w_{a}=0.4 \mathrm{~mm}, w_{p}=l_{p}=8.5 \mathrm{~mm}$; (a): $l_{a}=9.5 \mathrm{~mm}$, $l_{s}=1.67 \mathrm{~mm} ;(\mathrm{b}): l_{a}=8 \mathrm{~mm}, l_{s}=4.67 \mathrm{~mm}$.

which contains a ferrite layer biased in-plane. Note that the results of Fig. 8(a) and (b) are very similar to those shown in Fig. 6(a) and (b). In the measured antenna the metallic patch was printed on a thin dielectric layer, and this layer was placed on top of a ferrite sample of dimensions $0.625 \mathrm{~mm} \times 25 \mathrm{~mm}$ $\times 25 \mathrm{~mm}$. The ferrite sample employed is a low loss polycrystalline ytrium-iron-garnet (YIG) supplied by the Russian company Magneton (Magneton 9CHV's). This ferrite sample was separated from the ground planes containing the apertures by means of a foam layer (Rohacell 51). Apart from the three layers existing between the patch and the ground plane (the dielectric layer, the ferrite layer and the foam layer), a fourth additional air layer has been included in the simulations in order to account for the air gap existing between the ferrite layer and the dielectric

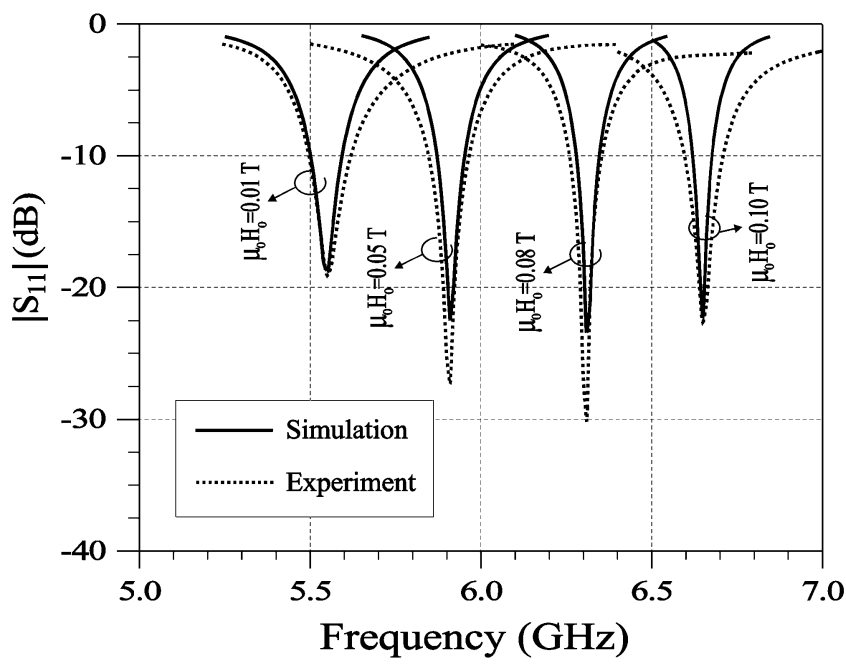

(a)

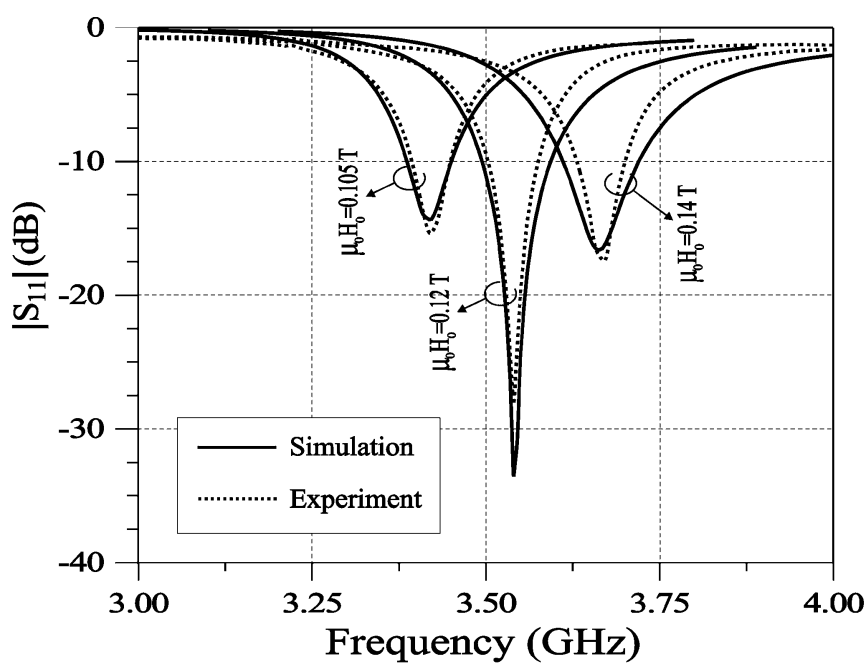

(b)

Fig. 8. Numerical and experimental results for the reflection coefficient of square microstrip patch coupled to a rectangular aperture [see Fig. 1(c)]. The patch lies on a four-layered substrate containing one ferrite layer. The ferrite is biased in-plane $\left(\theta_{0}=90^{\circ}, \phi_{0}=0^{\circ}\right)$. Results are presented for different values of the magnitude of the bias magnetic field $H_{0}$ above (a) and below (b) the REMW. (a) and (b): $h_{d 0}=0.635 \mathrm{~mm}, h_{d 1}=1.15 \mathrm{~mm}, h_{f}=$ $0.635 \mathrm{~mm}, h_{d 2}=0.01 \mathrm{~mm}, h_{d 3}=0.254 \mathrm{~mm}, \epsilon_{d 0}=10, \epsilon_{d 1}=1.07$, $\epsilon_{f}=15.3, \epsilon_{d 2}=1, \epsilon_{d 3}=10.5, \mu_{0} M_{s}=0.183 \mathrm{~T}, \mu_{0} \Delta H=0.0006 \mathrm{~T}$, $w_{s}=0.6 \mathrm{~mm}, w_{a}=0.4 \mathrm{~mm}, w_{p}=l_{p}=15.2 \mathrm{~mm}$; (a): $l_{a}=8.2 \mathrm{~mm}$, $l_{s}=6.2 \mathrm{~mm} ;(\mathrm{b}): l_{a}=12.5 \mathrm{~mm}, l_{s}=3 \mathrm{~mm}$.

layer supporting the patch. In Fig. 8(a) and (b) the agreement between theory and measurements is reasonably good. Both numerical and experimental results show that the antenna above the REMW can be tuned by about $20 \%$ as $\mu_{0} H_{0}$ is varied, and the antenna below the REMW can be tuned by about $7 \%$. As commented above, whereas the tuning range above the REMW is limited by the excitation of MSSWs, the tuning range below the REMW is limited by matching degradation as $\mu_{0} H_{0}$ recedes from $0.12 \mathrm{~T}$. The numerical bandwidth obtained for every value of $\mu_{0} H_{0}$ is slightly smaller than the experimental bandwidth above the REMW, and the opposite holds below the REMW. The experimental bandwidth lies around $2 \%$ both above and below the REMW. 


\section{CONCLUSION}

Multilayered printed antennas have been studied when one or more layers are magnetized ferrite materials. The bias magnetic field of the ferrites is allowed to be arbitrarily oriented. The algorithm is based on the application of the MoM in the spectral domain. The use of rooftop basis functions makes it possible to analyze a wide class of aperture coupled antennas with patches and apertures of different shapes. The results obtained show that it is possible to fabricate circularly and linearly polarized square patch antennas on ferrite substrates with a matching frequency band that can be tuned over a wide range by adjusting the magnitude of the bias magnetic field. Also, the authors have found that the polarization of a square patch antenna on ferrite substrate can be tuned from circular to linear within the same frequency band by changing the orientation and magnitude of the internal bias magnetic field. In the paper a new antenna has been designed which consists of stacked square patches on normally biased ferrite substrates. The new antenna provides wideband circular polarization and matching while requiring one single feed and a constant bias magnetic field. Finally, in order to check the validity of the numerical algorithm, some of the numerical results have been compared with measurements, and good agreement has been found.

\section{REFERENCES}

[1] D. M. Pozar and V. Sanchez, "Magnetic tuning of a microstrip antenna on a ferrite substrate," Electron. Lett., vol. 24, no. 12, pp. 729-731, 1988.

[2] H. How, P. Rainville, F. Harackiewicz, and C. Vittoria, "Radiation frequencies of ferrite patch antennas," Electron. Lett., vol. 28, no. 15, pp. 1405-1406, 1992.

[3] D. M. Pozar, "Radiation and scattering characteristics of microstrip antennas on normally biased ferrite substrates," IEEE Trans. Antennas Propag., vol. 40, pp. 1084-1092, Sep. 1992.

[4] H. How, T. M. Fang, D. X. Guan, and C. Vittoria, "Magnetic steerable ferrite patch antenna array," IEEE Trans. Magn., vol. 30, pp. 4551-4553, Nov. 1994.

[5] J. S. Roy, P. Vaudon, A. Reineix, F. Jecko, and B. Jecko, "Circularly polarized far fields of an axially magnetized circular ferrite microstrip antenna," Microwave Opt. Technol. Lett., vol. 5, no. 5, pp. 228-230, May 1992.

[6] K. K. Tsang and R. J. Langley, "Design of circular patch antennas on ferrite substrates," Proc. Inst. Elect. Eng. Microw. Antennas Propag., pt. H, vol. 145 , no. 1 , pp. 49-55, 1998.

[7] P. J. Rainville and F. J. Harackiewicz, "Magnetic tuning of a microstrip patch antenna fabricated on a ferrite film," IEEE Microwave Guided Wave Lett., vol. 2, pp. 483-485, Dec. 1992.

[8] H. How, T. M. Fang, and C. Vittoria, "Intrinsic modes of radiation in ferrite patch antennas," IEEE Trans. Microwave Theory Tech., vol. 42, pp. 988-994, Jun. 1994.

[9] T. Fukusako, A. Imahase, and N. Mita, "Polarization characteristics of patch antennas using in-plane and weakly biased ferrite substrate," IEEE Trans. Antennas Propag., vol. 52, no. 1, pp. 325-327, Jan. 2004.

[10] N. E. Buris, T. B. Funk, and R. S. Silverstein, "Dipole arrays printed on ferrite substrates," IEEE Trans. Antennas Propag., vol. 41, no. 2, pp. 165-175, Feb. 1993.

[11] H. How, T. M. Fang, W. Liu, C. Vittoria, M. H. Champion, and H. L. Southall, "Antenna array of circular patches on ferrite substrate," IEEE Trans. Magn., vol. 33, pp. 735-738, Jan. 1997.

[12] H. How, P. Shi, C. Vittoria, E. Hokanson, M. N. Champion, L. C. Kempel, and K. D. Trott, "Steerable phased array antennas using single-crystal YIG phase shifters-theory and experiments," IEEE Trans. Microwave Theory Tech., vol. 48, pp. 1544-1549, Sep. 2000.

[13] B. M. Cahill and J. C. Batchelor, "Electromagnetic scanning three element array with integral phase shifters," Electron. Lett., vol. 36, no. 18, pp. 1521-1522, 2000.

[14] H. Y. Yang, "Characteristics of switchable ferrite microstrip antennas," IEEE Trans. Antennas Propag., vol. 44, pp. 1127-1132, Aug. 1996.
[15] V. Losada, R. R. Boix, and F. Medina, "Evaluation of the radar cross section of circular microstrip patches on anisotropic and chiral substrates," IEEE Trans. Antennas Propag., vol. 49, no. 11, pp. 1603-1605, Nov. 2001.

[16] D. M. Pozar, "Microstrip antennas," Proc. IEEE, vol. 80, pp. 79-91, Jan. 1992.

[17] — "Microstrip antenna aperture-coupled to a microstripline," Electron. Lett., vol. 21, no. 2, pp. 49-50, 1985.

[18] P. L. Sullivan and D. H. Schaubert, "Analysis of an aperture coupled microstrip antenna," IEEE Trans. Antennas Propag., vol. 34, no. 8, pp. 977-984, Aug. 1986.

[19] F. Croq and A. Papiernik, "Large bandwidth aperture-coupled microstrip antenna," Electron. Lett., vol. 26, no. 16, pp. 1293-1294, Aug. 1990.

[20] F. Croq and D. M. Pozar, "Millimeter-wave design of wide-band aperture-coupled stacked microstrip antennas," IEEE Trans. Antennas Propag., vol. 39, no. 12, pp. 1770-1776, Dec. 1991.

[21] R. C. Hall and J. R. Mosig, "The analysis of arbitrarily shaped aperturecoupled patch antennas via a mixed-potential integral equation," IEEE Trans. Antennas Propag., vol. 44, no. 5, pp. 608-614, May 1996.

[22] S. D. Targonsky, R. B. Waterhouse, and D. M. Pozar, "Design of wide-band aperture-stacked patch microstrip antennas," IEEE Trans. Antennas Propag., vol. 46, no. 9, pp. 1245-1251, Sep. 1998.

[23] X. H. Yang and L. Shafai, "Characteristics of aperture coupled microstrip antennas with various radiating patches and coupling apertures," IEEE Trans. Antennas Propag., vol. 43, no. 1, pp. 72-78, Jan. 1995.

[24] H. F. Hammad, Y. M. M. Antar, and A. P. Freundorfer, "Dual band aperture coupled antenna using spur line," Electron. Lett., vol. 33, no. 25, pp. 2088-2090, 1997.

[25] D. M. Pozar and S. D. Targonski, "Improved coupling for aperture coupled microstrip antennas," Electron. Lett., vol. 27, no. 13, pp. 1129-1131, 1991.

[26] R. R. Boix, N. G. Alexopoulos, and M. Horno, "Efficient numerical computation of the spectral transverse dyadic Green's function in stratified anisotropic media," J. Electromagn. Waves Appl., vol. 10, no. 8, pp. 1047-1083, 1996.

[27] I. Y. Hsia, H. Y. Yang, and N. G. Alexopoulos, "Electromagnetically coupled dipole antennas in a nonreciprocal microstrip structure," in IEEE Antennas Propag. Soc. Symp. Dig., Chicago, IL, Jul. 1992, pp. 2220-2223.

[28] A. D. Brown, J. L. Volakis, L. C. Kempel, and Y. Y. Botros, "Patch antennas on ferromagnetic substrates," IEEE Trans. Antennas Propag., vol. 47, no. 1, pp. 26-32, Jan. 1999.

[29] M. I. Sobhy, M. W. R. Ng, R. J. Langley, and J. C. Batchelor, "TLM modeling of microstrip patch antenna on ferrite substrate," Int. J. RF Microwave CAE, vol. 11, no. 3, pp. 131-139, 2001.

[30] E. Drake, R. R. Boix, M. Horno, and T. K. Sarkar, "Effect of substrate dielectric anisotropy on the frequency behavior of microstrip circuits," IEEE Trans. Microwave Theory Tech., vol. 48, pp. 1394-1403, Aug. 2000.

[31] H. Y. Yang, J. A. Castaneda, and N. G. Alexopoulos, "Surface wave modes of printed circuits on ferrite substrates," IEEE Trans. Microwave Theory Tech., vol. MTT-40, pp. 613-621, Apr. 1992.

[32] D. M. Pozar, Microwave Engineering. Reading, MA: Addison-Wesley, 1990.

[33] T. Nurgaliev, S. Miteva, A. Jenkins, and D. Dew-Hughes, "Transmission characteristics of HTS microstrip resonators with a ferrite component," IEEE Trans. Appl. Supercond., vol. 11, pp. 446-449, Mar. 2001.

[34] M. Kahrizi, T. K. Sarkar, and Z. A. Maricevic, "Analysis of a wide radiating slot in the ground plane of a microstrip line," IEEE Trans. Microwave Theory Tech., vol. 41, no. 1, pp. 29-37, Jan. 1993.

[35] G. León, R. R. Boix, and F. Medina, "Full-wave analysis of a wide class of microstrip resonators fabricated on magnetized ferrites with arbitrarily oriented bias magnetic field," IEEE Trans. Microwave Theory Tech., vol. 50, no. 6, pp. 1510-1519, Jun. 2002.

[36] F. L. Mesa, R. Marqués, and M. Horno, "A general algorithm for computing the bidimensional spectral Green's dyad in multilayered complex bianisotropic media: The equivalent boundary method," IEEE Trans. Microwave Theory Tech., vol. 39, no. 9, pp. 1640-1649, Sep. 1991.

[37] R. E. Collin, Antennas and Radiowave Propagation. New York: McGraw-Hill, 1985.

[38] G. León, R. R. Boix, and F. Medina, "Efficient full-wave characterization of microstrip lines fabricated on magnetized ferrites with arbitrarily oriented bias field," J. Electromagn. Waves Appl., vol. 15, no. 2, pp. 223-252, 2001.

[39] M. S. Sodha and N. C. Srivastava, Microwave Propagation in Ferrimag netics. New York: Plenum, 1981. 
[40] V. Losada, R. R. Boix, and M. Horno, "Full-wave analysis of circular microstrip resonators in multilayered media containing uniaxial anisotropic dielectrics, magnetized ferrites and chiral materials," IEEE Trans. Microwave Theory Tech., vol. 48, no. 6, pp. 1057-1064, Jun. 2000.

[41] R. B. Waterhouse, "Stacked patches using high and low dielectric constant material combinations," IEEE Trans. Antennas Propag., vol. 47, no. 12, pp. 1767-1771, Dec. 1999.

[42] T. Vlasits, E. Korolkiewicz, A. Sambell, and B. Robinson, "Performance of a cross-aperture coupled single feed circularly polarized patch antenna," Electron. Lett., vol. 32, no. 7, pp. 612-613, 1996.

[43] S. D. Targonski and D. M. Pozar, "Design of wideband circularly polarized aperture-coupled microstrip antennas," IEEE Trans. Antennas Propag., vol. 41, no. 2, pp. 214-220, Feb. 1993.

[44] R. R. El-Idrissi, R. Marqués, and F. Medina, "Comprehensive analysis of strip-line and slot-line guided forward, backward and complex magnetostatic waves," IEEE Trans. Microwave Theory Tech., vol. 49, no. 9, pp. 1599-1606, Sep. 2001.

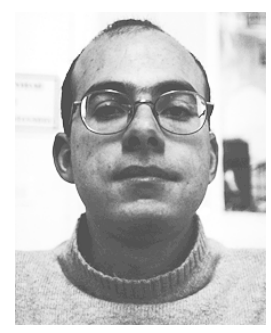

Germán León was born in Alcázar de San Juan, Spain, in 1975. He received the Licenciado and Ph.D. degrees in physics from the University of Seville, Sevilla, Spain, in 1998 and 2005, respectively.

His research interests focus on the effect of ferrite substrates on the performance of planar passive microwave circuits and printed circuit antennas.

Dr. León received a grant from the Spanish Ministry of Education and Culture to support his research toward the Ph.D. degree in 1999.

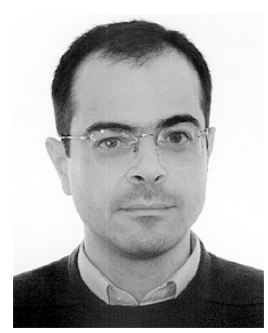

Rafael R. Boix (M'96) received the Licenciado and $\mathrm{Ph} . \mathrm{D}$. degrees in physics from the University of Seville, Sevilla, Spain, in 1985 and 1990 respectively.

Since 1986, he has been with the Electronics and Electromagnetism Department, University of Seville, where he became Associate Professor in 1994. During the summers of 1991 and 1992, he was with the Electrical Engineering Department, University of California at Los Angeles, as a Visiting Scholar. During summer 1996, he was with the Electrical and Computer Engineering Department, Syracuse University, Syracuse, NY, as a Visiting Scholar. His current research interests include the study of microstrip circuits and antennas on ferrite substrates, and the analysis and design of periodic electromagnetic structures such as electromagnetic bandgap transmission lines and frequency selective surfaces.

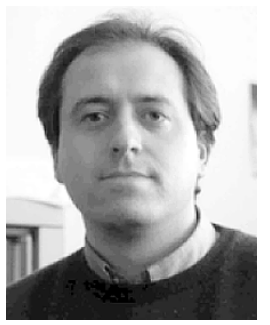

Manuel J. Freire was born in Cádiz, Spain, in 1972. He received the Licenciado and Ph.D. degree in physics from the University of Seville, Sevilla, Spain, in 1995 and 2000, respectively.

From 1995 to 1996, he served in the Royal Naval Observatory of the Spanish Navy, San Fernando, Spain. In 1996, he joined the Microwave Group of the Department of Electronics and Electromagnetism, University of Seville. In 1998 he was a Visiting Scholar in the Department of Electrical and Computer Engineering, University of Houston, TX. His research interests include leakage in microstrip lines, effect of ferrites on microstrip devices and metamaterials. He is currently an Assistant Professor in the Department of Electronics and Electromagnetism, University of Seville.

Dr. Freire was the recipient of a grant from the Spanish Ministry of Education to support his research toward the Ph.D. degree.

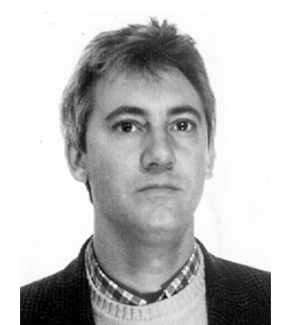

Francisco Medina (M'90-SM'01) received the Licenciado and $\mathrm{Ph} . \mathrm{D}$. degrees in physics from the University of Seville, Sevilla, Spain, in 1983 and 1987 respectively.

Since 1983, he has been with the Electronics and Electromagnetism Department, University of Seville, where he became Associate Professor in 1990. He is currently the head of the Microwaves Group at the same Department. Between 1986 and 1987, he spent the academic year at the Laboratoire de Microondes of L'ENSEEIHT, Toulouse, France. His research interest include analytical and numerical methods for guiding, resonating and radiating structures, passive planar circuits, periodic structures, and the influence of anisotropic materials (including microwave ferrites) on such structures. $\mathrm{He}$ is also interested in artificial media modeling and design.

Dr. Medina was a Member of the Technical Programme Committees (TPC) of the 23rd European Microwave Conference (Madrid, Spain, 1993), the ISRAMT'99 (Málaga, Spain, 1999), and a member of the TPC of the Microwaves Symposium'2000 (Tetouan, Morocco, 2000). He was co-organizer of the workshop New Trends on Computational Electromagnetics for Open and Boxed Microwave Structures, Madrid, Spain, 1993. He has acted as a reviewer for several IEEE journals, for journals of the Institution of Electrical Engineers, London (IEE) U.K., and for journals of the American Physics Association. 